\title{
Correction to: Absorption of calcium ions on oxidized graphene sheets and study its dynamic behavior by kinetic and isothermal models
}

\author{
Mahmoud Fathy ${ }^{1} \cdot$ Th. Abdel Moghny ${ }^{1} \cdot$ Mahmoud A. Mousa $^{2} \cdot$ Moaz M. Abdou $^{3} \cdot$ Abdel-Hameed A-A. El-Bellihi $^{2}$. \\ Ahmed E. Awadallah ${ }^{3}$
}

Published online: 29 September 2018

○) Springer-Verlag GmbH Germany, part of Springer Nature 2018

\section{Correction to: Appl Nanosci (2016) 6:1105-1117 https://doi.org/10.1007/s13204-016-0537-8}

In the original publication, the author group has been published incorrectly. The correct author group is given below.

Mahmoud Fathy ${ }^{1}$, Th. Abdel Moghny ${ }^{1}$, Mahmoud A. Mousa $^{2}$, Moaz M. Abdou ${ }^{3}$, Abdel-Hameed A-A. El-Bellihi' ${ }^{2}$, Ahmed E. Awadallah ${ }^{3}$

${ }^{1}$ Applications Department, Egyptian Petroleum Research Institute (EPRI), Cairo, Egypt

${ }^{2}$ Faculty of Science, Benha University, Cairo, Egypt

${ }^{3}$ Development of Processes Department, Egyptian Petroleum Research Institute, Cairo, Egypt

The original article can be found online at https://doi.org/10.1007/ s13204-016-0537-8.

Mahmoud Fathy

fathy8753@yahoo.com

1 Applications Department, Egyptian Petroleum Research Institute (EPRI), Cairo, Egypt

2 Faculty of Science, Benha University, Cairo, Egypt

3 Development of Processes Department, Egyptian Petroleum Research Institute, Cairo, Egypt 